\section{Información sobre prevención de enfermedades respiratorias en trabajadores de la construcción}

\author{
Information on respiratory disease prevention in construction \\ workers
}

\author{
Informações sobre prevenção de doenças respiratórias em \\ trabalhadores da construção civil
}

\author{
José Ostos \\ jfrancisco.92@gmail.com
}

\begin{abstract}
RESUMEN
Introducción: Entre las enfermedades más comunes en el ambiente laboral se tienen las de origen respiratorio, que ocasionan una interrupción en el intercambio gaseoso, debido a una mayor interacción con los agentes ambientales, capaces de provocar casi todos los tipos de enfermedades pulmonares crónicas. Objetivo: Identificar la información que tienen los trabajadores de la construcción de una institución educativa, acerca de la prevención de las enfermedades respiratorias para el primer trimestre del año 2016. Materiales y Métodos: Investigación descriptiva, con un diseño de campo de corte transversal y contemporáneo; la población objeto de estudio estuvo conformada por veinte (20) trabajadores obreros que laboran en una institución educativa. Para recolectar la información se utilizó como técnica la encuesta, y el instrumento fue el CISRT (Cuestionario de Información sobre Riesgos Respiratorios en el Trabajo). Resultados y Discusión: Los resultados dan cuenta que un alto porcentaje de los trabajadores posee información sobre aspectos generales de las enfermedades respiratorias referidos a tipo de enfermedad, causas, síntomas, complicaciones y diagnóstico; no obstante, otro grupo responde de forma incorrecta acerca de la prevención. Conclusión: Se recomienda promover la salud ocupacional a través sesiones educativas, acerca de las generalidades de las enfermedades respiratorias ocasionadas por el trabajo en la construcción y las medidas de prevención tanto en la fuente primaria, en el ambiente como en el propio trabajador.
\end{abstract}

Palabras clave: enfermedades respiratorias; enfermedades profesionales; trabajadores

\begin{abstract}
Introduction: Among the most common diseases in the work environment are those of respiratory origin, which cause an interruption in the gas exchange, due to a greater interaction with environmental agents, capable of causing almost all types of chronic lung diseases. Objective: To identify the information that construction workers have in an educational institution, about the prevention of respiratory diseases for the first quarter of 2016. Materials and Methods: Descriptive research, with a cross-sectional and contemporary field design. The population under study was made up of twenty (20) workers who work in an educational institution. To collect the information, the survey was used as a technique, and the instrument was the CISRT (Information Questionnaire on Respiratory Risks at Work). Results and Discussion: The results show that a high percentage of workers have information on general aspects of respiratory diseases related to type of disease, causes, symptoms, complications and diagnosis; however, another group responds incorrectly about prevention. Conclusion: It is recommended to promote occupational health through educational sessions, about the generalities of respiratory diseases caused by construction work and prevention measures both at the primary source, in the environment and in the worker himself.
\end{abstract}

J0: Universidad Central de Venezuela, Venezuela

Key words: respiratory diseases; occupational diseases; workers 
J0: Universidad Central de Venezuela, Venezuela

\section{RESUMO}

Introdução: Dentre as doenças mais comuns no ambiente de trabalho estão as de origem respiratória, que causam interrupção nas trocas gasosas, devido a uma maior interação com agentes ambientais, capazes de causar quase todos os tipos de doenças pulmonares crônicas. Objetivo: Identificar as informações que os trabalhadores da construção civil possuem em uma instituição de ensino sobre prevenção de doenças respiratórias no primeiro trimestre de 2016. Materiais e Métodos: Pesquisa descritiva, com desenho de campo transversal e contemporâneo. ; A população estudada foi composta por vinte (20) trabalhadores que trabalham em uma instituição de ensino. Para a coleta das informações, utilizou-se a pesquisa como técnica e o instrumento foi o CISRT (Questionário de Informações sobre Riscos Respiratórios no Trabalho). Resultados e Discussão: Os resultados mostram que uma alta porcentagem de trabalhadores possui informações sobre aspectos gerais das doenças respiratórias relacionadas ao tipo de doença, causas, sintomas, complicações e diagnóstico; no entanto, outro grupo responde incorretamente sobre prevenção. Conclusão: Recomenda-se promover a saúde ocupacional, por meio de sessões educativas, sobre as generalidades das doenças respiratórias causadas pelas obras e medidas de prevenção, tanto na fonte primária, no meio ambiente quanto no próprio trabalhador.

Palavras-chave: doenças respiratórias; doenças ocupacionais; trabalhadores

\section{INTRODUCCIÓN}

$\mathrm{E}$ l origen de las enfermedades está relacionado con las costumbres, la alimentación, el descanso, y/o el medio ambiente en el cual el ser humano se desenvuelve y donde pasa la mayor parte de su tiempo productivo. En el ámbito laboral, las enfermedades están relacionadas con la actividad misma, los medios de trabajo, la interacción que hace el trabajador con los medios de trabajo, el entorno y la organización, en el cual la persona se encuentra obligada a trabajar, los cuales son factores que intervienen en la salud del trabajador.

Es indispensable conceder importancia a la salud ocupacional, como la ciencia de la anticipación, reconocimiento y evaluación de riesgos nocivos en el ambiente de trabajo, así como del desarrollo de estrategias de prevención y control, con el objetivo de proteger y promover la salud y el bienestar de los trabajadores, salvaguardando también la comunidad y el medio ambiente en general (1). Desde esta área, se busca fundamentalmente la prevención de accidentes y enfermedades ocupacionales.
Según datos de la Organización Mundial de la Salud (OMS) y la Organización Internacional del Trabajo (OIT) indican, que el riesgo de contraer una enfermedad profesional se ha convertido en el peligro más frecuente al que se enfrentan los trabajadores en sus empleos. Estas enfermedades causan anualmente unos 1,7 millones de muertes relacionadas con el trabajo y superan a los accidentes mortales en una proporción de cuatro a uno (2).

Los accidentes y la aparición de enfermedades son uno de los aspectos más alarmantes en el ambiente laboral. Entre las enfermedades más comunes en el ambiente laboral se tienen, las de origen músculo-esqueléticas, debido a la presencia de traumatismos por caídas o por una inadecuada mecánica corporal y también, las de origen respiratorio, que ocasionan una interrupción en el intercambio gaseoso, debido a una mayor interacción con los agentes ambientales, entre otros. Se ha calculado que en un trabajo de 40 horas semanales, se introducen unos 14.000 litros de aire en las vías aéreas. Las sustancias inhaladas durante ese tiempo, son capaces de 
provocar casi todos los tipos de enfermedades pulmonares crónicas (3).

Cuando las enfermedades

respiratorias de origen ocupacional no son tratadas a tiempo, se pueden originar graves complicaciones en las personas afectadas, que pueden variar desde una enfermedad pulmonar obstructiva crónica (EPOC) hasta un cáncer pulmonar, esto se traduce en disminución de la calidad de vida del trabajador: sufrimiento, costos económicos para él y su familia, ausentismo laboral y en el peor de los casos, la muerte prematura por una causa perfectamente prevenible.

Entre las patologías ocupacionales que afectan al sistema respiratorio se pueden mencionar: asma, bronquitis, bronquiolitis, neumonitis por hipersensibilidad, silicosis y neumoconiosis. Respecto de la silicosis, un estudio realizado por el Instituto de Salud Pública de Chile, estimó que el 5,4\% de los trabajadores, en el año 2008, estaban expuestos a sílice (4). La construcción es una de las áreas que más riesgo produce para este tipo de enfermedad, sin embargo, cualquier actividad en la que se fracture roca o se trabaje con este elemento, puede generar silicosis. El número real de expuestos está, muy probablemente, subestimado.

Un colectivo trabajador expuesto a las enfermedades respiratorias ocupacionales es el de la construcción civil, que son todos aquellos que realizan una labor de construcción para otra persona, ya sea natural o jurídica, en relación de dependencia y a cambio de una remuneración. Para Weeks (5) un alto porcentaje de trabajadores de la construcción, son no cualificados y los obreros engloban del 5 al $10 \%$ de la población activa", con lo cual tienen poca formación y/o escolaridad que hace inferir el poco conocimiento sobre prevención de riesgos en su oficio. Este autor expresa, que los trabajadores de la construcción también están clasificados por oficios especializados, por ejemplo: albañiles, carpinteros, electricistas, cristaleros, pintores, fontaneros, excavadores de túneles, trabajadores de mantenimiento, entre otros.

En el sector de la construcción, se ha observado incremento de enfermedades de tipo respiratorio debido a la exposición continua a polvos, material particulado, condiciones de trabajo inadecuadas, ausencia de sistemas de control a nivel de la fuente, del ambiente o del trabajador, la falta de información que se tiene acerca de la prevención y a su vez, los empleadores no actúan apegados a la normativa nacional e internacional en materia de salud ocupacional (6).

Para la Organización Internacional del Trabajo (OIT), muchos trabajadores fallecen en el lugar de trabajo cada año y los trabajadores de la construcción, tienen una probabilidad entre 3 y 4 veces mayor de morir, a causa de accidentes en el trabajo que otros trabajadores. Los riesgos derivados de la exposición a los variados agentes en el campo de la construcción, pueden ser de 3 a 6 veces mayores (3).

La Oficina de Estadísticas de la Unión Europea (EUROSTAT) para el año 2003, señaló que los trastornos respiratorios eran la tercera enfermedad más común entre los problemas de salud relacionados con el trabajo, particularmente en las industrias mineras. Este autor también señala que para el año 2006, el Instituto Nacional de Estadísticas (INE) de Chile, indicó que 3.881 hombres y 3.810 mujeres pertenecientes a la clase trabajadora de ese país, fallecieron por trastornos del sistema respiratorio (6).

En Venezuela, según el Instituto Nacional de Prevención, Salud y Seguridad Laborales (INPSASEL) para el año 2012, establece que el $7,0 \%$ de la población trabajadora en el sector de la construcción se vio afectada (7). Debido al crecimiento 
poblacional en el país, se ha dado la necesidad de realizar grandes construcciones de origen habitacional como por ejemplo, la Gran Misión Vivienda Venezuela o urbanizaciones privadas, así como también, construcciones de origen comercial para el entretenimiento de la población u otros y con ello, el incremento de trabajadores que se dediquen al área de la construcción, que debido a la falta de información que se tiene acerca de las enfermedades respiratorias $\mathrm{y}$ sus complicaciones y el inadecuado uso de los métodos de barrera o dispositivos de protección personal, se ha visto un incremento en cuanto a patologías respiratorias. Esto es un problema para la familia, el propio trabajador, las empresas, así como para las instituciones de salud. El hecho que los trabajadores de la construcción no posean información sobre la prevención de las enfermedades respiratorias, trae como consecuencia, no solo un costo económico, sino que también ocasiona un costo social ya que altera el núcleo familiar, las actividades del trabajador y también produce un impacto psicológico.

Estos trabajadores de la construcción, realizan múltiples labores con materiales: bloques, cerámicas, granito, porcelanato, mármol, entre otros; también están expuestos a una cantidad de material particulado, como lo son, polvos provenientes del mismo cemento o la piedra. Cabe destacar, que en la población objeto de estudio, se ha observado que no siempre hacen uso de los equipos de protección personal, situación ésta, que a mediano y a largo plazo puede influir en la aparición de enfermedades respiratorias.

Es por ello que el objetivo de la presente investigación, es identificar la información que tienen los trabajadores de la construcción de una institución educativa, acerca de la prevención de las enfermedades respiratorias para el primer trimestre del año 2016. La plantilla de obreros en estudio realiza permanentemente grandes trabajos de construcción, pues la institución se encuentra en expansión.

\section{MATERIALES Y MÉTODOS}

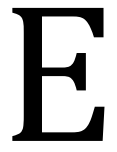
sta investigación se adscribe al tipo de estudio descriptivo, con un diseño de campo, transversal y contemporáneo. La población-objeto de estudio, estuvo conformada por 20 trabajadores que laboran en esta institución y por ser una población pequeña, se decidió trabajar con la totalidad. El método seleccionado para la recolección de datos fue la encuesta, cuyo instrumento utilizado fue el cuestionario el CISRT (Cuestionario de Información sobre Riesgos Respiratorios en el Trabajo), diseñado con preguntas dicotómicas. En cuanto a la confiabilidad del instrumento, se utilizó la prueba Alfa de Cronbach, donde se obtuvo un resultado de 0.7 , por lo tanto, se consideró que el instrumento era lo suficientemente confiable. Finalmente, se recolectó la información previo acuerdo con los trabajadores, en el marco de los principios éticos que rigen toda investigación.

\section{RESULTADOS}

$\mathrm{P}$ osterior a la recolección de la información obtenido del CISRT, se procedió a procesar la información, obteniendo los siguientes resultados: 
Tabla 1. Información que poseen los trabajadores de la construcción acerca de los aspectos generales de las enfermedades respiratorias

\begin{tabular}{lllllll}
\hline \multicolumn{1}{c}{ Ítem } & \multicolumn{2}{c}{ CORRECTA } & \multicolumn{2}{c}{ INCORRECTA } & \multicolumn{2}{c}{ TOTAL } \\
& Fa & \% & Fa & \% & Fa & \% \\
\hline Enfermedad ocupacional & 19 & 95 & 1 & 5 & 20 & 100 \\
Enfermedad respiratoria & 20 & 100 & 0 & 0 & 20 & 100 \\
Enfermedad en el trabajo & 4 & 20 & 16 & 80 & 20 & 100 \\
Asma & 14 & 70 & 6 & 30 & 20 & 100 \\
Silicosis & 8 & 40 & 4 & 20 & 20 & 100 \\
Causas de enfermedad & 19 & 95 & 1 & 5 & 20 & 100 \\
respiratoria & 16 & 80 & 4 & 20 & 20 & 100 \\
Signos y síntomas & 20 & 100 & 0 & 0 & 20 & 100 \\
Complicaciones & 20 & 100 & 0 & 0 & 20 & 100 \\
Diagnósticos & & & & & & \\
\hline
\end{tabular}

Los resultados de la tabla 1, enfatizan que un alto porcentaje de la población encuestada, posee información sobre las enfermedades respiratorias ocupacionales, los signos y síntomas, complicaciones y diagnóstico; es de notar, que con respecto a la silicosis, el $60 \%$ respondió de forma incorrecta y sobre el asma, un $30 \%$ respondió de forma incorrecta. Destaca una incongruencia, pues responde la mayoría de forma acertada a la pregunta sobre enfermedad ocupacional, pero no, cuando se indaga sobre enfermedades del trabajo.

Tabla 2. Información que poseen los trabajadores de la construcción acerca de la prevención de las enfermedades respiratorias

\begin{tabular}{|c|c|c|c|c|c|c|}
\hline \multirow[t]{2}{*}{ Ítem } & \multicolumn{2}{|c|}{ CORRECTA } & \multicolumn{2}{|c|}{ INCORRECTA } & \multicolumn{2}{|c|}{ TOTAL } \\
\hline & $\mathbf{F a}$ & $\%$ & $\mathbf{F a}$ & $\%$ & $\mathbf{F a}$ & $\%$ \\
\hline Sustitución del agente tóxico & 7 & 35 & 13 & 65 & 20 & 100 \\
\hline $\begin{array}{l}\text { Sustitución de método por menor } \\
\text { riesgo }\end{array}$ & 4 & 20 & 16 & 80 & 20 & 100 \\
\hline $\begin{array}{l}\text { Sistema de ventilación } \\
\text { humidificación }\end{array}$ & 13 & 65 & 7 & 35 & 20 & 100 \\
\hline Org. puesto de trabajo & 6 & 30 & 14 & 70 & 20 & 100 \\
\hline Exámenes médicos & 3 & 15 & 17 & 85 & 20 & 100 \\
\hline Equipos de protección personal & 20 & 100 & 0 & 0 & 20 & 100 \\
\hline
\end{tabular}

En la tabla 2, se aprecia que los obreros encuestados, responden de forma correcta acerca de los sistemas de ventilación-humidificación, como medida de prevención y sobre los equipos de protección. No obstante, con respecto a la medida de prevención primaria de sustituir el agente tóxico por métodos de menor riesgo, organización del puesto de trabajo y exámenes médicos, hubo un alto porcentaje que respondió de forma incorrecta. 


\section{DISCUSIÓN}

$\mathrm{L}$ os trabajadores de la construcción, en su mayoría, poseen información acerca de las generalidades de las enfermedades respiratorias. Esto se puede evidenciar en aquellos ítems en donde se les pregunta: ¿Qué es una enfermedad ocupacional y respiratoria, sus causas, así como sus signos y síntomas, complicaciones y diagnósticos? Así mismo, un alto porcentaje de esta población, desconoce cuáles son las enfermedades ocupacionales presentes en el lugar de trabajo, así como el concepto de asma ocupacional y silicosis, ambas enfermedades de tipo respiratorias y a su vez ocupacionales, presentes en el campo de la construcción. En el ítem 3, relacionado a las enfermedades ocupacionales presentes en el lugar de trabajo, el $80 \%$ respondió de forma incorrecta. Las enfermedades ocupacionales de tipo respiratorias de mayor incidencia, que están presentes en el campo de la construcción son: Asma, silicosis y Enfermedad Pulmonar Obstructiva Crónica (EPOC). El 30\% de estos trabajadores, desconocen el origen del asma ocupacional y el $60 \%$ de esta población, no está familiarizado con el concepto de silicosis, una enfermedad presente en el campo de la construcción.

Los ítems que conforman la tabla 2, están relacionados a la información que manejan estos trabajadores, acerca de la prevención de las enfermedades respiratorias en el campo de la construcción. Se puede definir prevención en el área de la salud, como "aquellas medidas o acciones que van encaminadas a promover el bienestar del ser humano, evaluando los factores de riesgos presentes en este y así evitar que un evento, llámese accidente o enfermedad, produzca daños irreversibles en la salud del mismo". La población de estudio, desconoce cómo se debe realizar la prevención efectiva de las enfermedades en su campo de trabajo. En lo que a sustitución de agentes tóxicos se refiere, un $65 \%$ de la población desconoce de la misma. Weeks (5) señala que "ciertos agentes en el campo de la construcción pueden ser sustituidos por otros, de tal forma de proteger a los trabajadores". El 80\% de esta población, expresó de forma incorrecta el ítem de sustitución de métodos de mayor riesgo, por otros de menor riesgo. Se ha referido en investigaciones, que algunos equipos o máquinas utilizados en el campo de la construcción, pueden ser sustituidos por otros, para así controlar los diferentes factores de riesgo y evitar la aparición de enfermedades ocupacionales en estos trabajadores (5).

En los trabajos de construcción, deben existir los controles medioambientales, que no solo favorece a disminuir a la contaminación del ambiente, sino que ayuda en lo que es la prevención de enfermedades respiratorias, sean de origen ocupacional o no. Para los controles medioambientales, se hace uso de los sistemas de humidificación o ventilación. El 35\% de la población de esta institución, desconoce acerca de ello. Se ha descrito que los controles medioambientales, se utilizan para eliminar una sustancia peligrosa del entorno o para protegerse de la fuente (3).

En el ítem relacionado a la organización de los puestos de trabajos, se observa que estos trabajadores desconocen acerca de ello, ya que un $70 \%$ respondió de forma incorrecta. Aunque se detecta falta de información acerca de la prevención por parte de estos trabajadores, los mismos poseen información acerca de cuáles son los equipos de protección personal que deben usar en este campo de trabajo, un 100\% contestó de forma correcta. 
La capacitación constante del trabajador en su sitio de labores, constituye en sí misma, una medida para evitar accidentes y enfermedades ocupacionales. Es una estrategia deseable para facilitar el cambio y permitir que el personal de la empresa, participe de forma activa en la prevención de enfermedades en el trabajo (8).

Riaño (9) expresa en su investigación, el predominio existente de patologías respiratorias. De allí extrae, que se evidencia la prevención y educación al trabajador, debido al riesgo atribuible poblacional, que según la investigadora, se ha estimado en un $15 \%$ en relación con la exposición ocupacional para adquirir una patología del sistema respiratorio, como enfermedad pulmonar obstructiva o asma de origen ocupacional.

Se hace necesario, reforzar la información a través de diferentes sesiones educativas con un personal de salud calificado, inspección al puesto de trabajo para verificar las condiciones y orientar a los trabajadores en plena actividad, efectuar actividades de sensibilización hacia la prevención, este es un trabajo continuo, también debe fomentarse la creación del comité de Higiene y Seguridad Laboral. Al respecto Loro (10), expresa que es necesario hacer hincapié en realizar actividades educativas continuamente, ya que es posible examinar las situaciones que causan peligro y su impacto en la vida de los trabajadores, mediante las acciones de higiene del trabajo, con el objetivo de evitar los accidentes y enfermedades y en consecuencia mejorar la salud de los trabajadores.

\section{CONCLUSIONES}

A manera de conclusión, se puede decir entonces, que los trabajadores de la construcción objeto del presente estudio, presentan un nivel de instrucción bajo y muy pocos son técnicos superiores, sin embargo, manejan información con respecto a aspectos generales de las enfermedades respiratorias. A pesar de poseer esta información, no saben identificar las diferentes enfermedades respiratorias de origen ocupacional que se presentan en el campo de la construcción, como el asma y la silicosis.

La cultura preventiva tiene un papel importante dentro de las organizaciones y debe ser vista de forma integral, así se considera no sólo el área de seguridad, sino también salud, ambiente, higiene, ergonomía, entre otras. El establecimiento de políticas preventivas es prioritario y el trabajador es el elemento primordial para lograrla (11).

- Conflicto de intereses: Ninguno declarado por el autor.

- Financiación: Autofinanciado.

- Agradecimientos: Ninguno declarado por el autor.

\section{REFERENCIAS BIBLIOGRÁFICAS}

1. Sánchez A. Enfermedades potenciales derivadas de factores de riesgo presentes en la industria de producción de alimentos. Octubre - diciembre 2011. Med Segur. Trab. [Internet] 2011; 57 (225) 300-312 Disponible en: https://pdfs.semanticscholar.org/2440 /03285a237a61c4fae0e543b983d06f1f a17e.pdf

2. Organización Mundial de la Salud. El número de accidentes y enfermedades relacionados con el trabajo sigue aumentando. [Internet]. 2017. Consultado 25/07/2017. Disponible en: http://www.who.int

3. Organización Internacional del Trabajo. La construcción un trabajo peligroso [citado 201726 de septiembre].Disponible en: https://www.ilo.org/global/topics/safetyand-health-atwork/areasofwork/hazardouswork/WCMS_356582/lang--es/index.htm 
4. Contreras G, Torrealba B. Enfermedades respiratorias ocupacionales. Rev. chil. Enferm. respir. [Internet]. 2013 [citado 201821 de julio]; 29(4): 189190.doi.org/10.4067/S071773482013000400001.

5. Weeks J. Enciclopedia de la OIT. Vol III. España: OIT; 2001. Disponible en: http://www.insht.es/
6. Farmer, G. Epidemiología de las enfermedades profesionales. Gobierno de Chile. 2012. [Internet]. Disponible en: http//www.sigweb.cl/

7. Instituto Nacional de Prevención, Salud y Seguridad Laboral (INPSASEL). Accidentes laborales formalizados ante INPSASEL. 2012. Caracas, Venezuela. [Internet] Disponible en: http://www.inpsasel.gob.ve/ 